\title{
Chaotic Behavior of One-Dimensional Cellular Automata Rule 24
}

\author{
Zujie Bie, ${ }^{1}$ Qi Han, ${ }^{2}$ Chao Liu, ${ }^{2}$ Junjian Huang, ${ }^{3}$ Lepeng Song, ${ }^{2}$ and Yangjun Pei ${ }^{2}$ \\ ${ }^{1}$ Internet Data Center, Chongqing University of Science and Technology, Chongqing 401331, China \\ ${ }^{2}$ School of Electrical and Information Engineering, Chongqing University of Science and Technology, Chongqing 401331, China \\ ${ }^{3}$ Department of Mathematics and Information Engineering, Chongqing University of Education College, Chongqing 400065, China
}

Correspondence should be addressed to Qi Han; hanqicq@163.com

Received 21 January 2014; Accepted 11 April 2014; Published 15 May 2014

Academic Editor: Zhen Jin

Copyright (c) 2014 Zujie Bie et al. This is an open access article distributed under the Creative Commons Attribution License, which permits unrestricted use, distribution, and reproduction in any medium, provided the original work is properly cited.

\begin{abstract}
Wolfram divided the 256 elementary cellular automata rules informally into four classes using dynamical concepts like periodicity, stability, and chaos. Rule 24 , which is Bernoulli $\sigma_{\tau}$-shift rule and is member of Wolfram's class II, is said to be simple as periodic before. Therefore, it is worthwhile studying dynamical behaviors of four rules, whether they possess chaotic attractors or not. In this paper, the complex dynamical behaviors of rule 24 of one-dimensional cellular automata are investigated from the viewpoint of symbolic dynamics. We find that rule 24 is chaotic in the sense of both Li-Yorke and Devaney on its attractor. Furthermore, we prove that four rules of global equivalence $\varepsilon_{5}^{2}$ of cellular automata are topologically conjugate. Then, we use diagrams to explain the attractor of rule 24 , where characteristic function is used to describe the fact that all points fall into Bernoulli-shift map after two iterations under rule 24.
\end{abstract}

\section{Introduction}

Cellular automata (CA) was first introduced by von Neumann in 1951 [1]. CA is a mathematical model consisting of large numbers of simple identical components with local interactions [2]. The simple components act together to produce complex global behavior. CA performs complex computation with high degree of efficiency and robustness. Three major factors have resulted in the revival of interest in the behavior of cellular systems [3]. First, the development of powerful computers and microprocessors has made the rapid simulation of CA possible. Second, the use of CA to simulate physical systems has attracted much interest in the scientific community. Third, the advent of VLSI as an implementation medium has focused attention on the communication requirements of successful hardware algorithms. Therefore, many researches about CA have been reported [46], especially in cryptography [7-9] and image processing $[10,11]$. In addition, the analysis of dynamical behavior about dynamical system has aroused wide public concern [12-16], such as chaotic behavior $[17,18]$. Thus, it is also important to analyze chaotic behavior of CA.
Here, we will only consider Boolean automata for which the cellular state $x \in\{0,1\}$. A cellular automaton consists of a number of cells which evolve by a simple local rule (identical rule). The value of each cell in the next stage is determined by the values of the cell and its neighbor cells in the current stage under the local rule [19]. The identical rule contained in each cell is essentially a finite state machine (FSM) which is specified in the form of a rule table [20]. A rule table contains an entry for possible neighborhood which consists of a cell and the adjacent cells. The cellular array is $d$-dimensional, where $d=1,2,3$ is used in practice. In this paper, we will concentrate on $d=1$. For a one-dimensional CA, a cell is connected to $r$ local neighbors (cells) on either side, where $r$ is referred to as the radius. A one-dimensional CA has $n$ cells linked in a line or in a circle. Denote the value in the $i$ th cell at the $t$ th stage by $x_{i}[t]$. For 2-state 3 -neighborhood CA $(r=1)$, the evolution of $i$ th cell can be represented as a function of the present states of $(i-1)$ th, $(i)$ th, and $(i+1)$ th. The local function $f_{i}$ is a deterministic function to determine the nextstage value of the $i$ th cell, $x_{i}[t+1]=f_{i}\left(x_{i-1}[t], x_{i}[t], x_{i+1}[t]\right)$. For example, the rule 24 is a one-dimensional CA and its rule table is shown in Table 1 . Thus, we have $f(000)=0$, 
TABLE 1: The truth table of Boolean function of rule 24 .

\begin{tabular}{lccc}
\hline$x_{i-1} x_{i} x_{i+1}$ & {$\left[f_{24}(x)\right]_{i}$} & $x_{i-1} x_{i} x_{i+1}$ & {$\left[f_{24}(x)\right]_{i}$} \\
\hline 000 & 0 & 100 & 1 \\
001 & 0 & 101 & 0 \\
010 & 0 & 110 & 0 \\
011 & 1 & 111 & 0 \\
\hline
\end{tabular}

$f(001)=0, f(010)=0, f(011)=1, f(100)=1, f(101)=0$, $f(110)=0$, and $f(111)=0$. Then, we find that the groups of three bits between parentheses represent all the possible neighborhood states and the single bit after equal sign is the resulting output bit at the next time step. The rule number is obtained by multiplying each output bit by the corresponding power of two and adding the results. Therefore, there exist $2^{8}=256$ possible rules with $r=1$. In this paper, we mainly discuss rule 24 . The rule 24 can be used to extract binary image edges. We know that bit strings 011 and 100 must be detected at the edges of binary images. If every row of an image is evolved according to the rule 24, every column of the image is evolved according to the rule 24 and their results do bitwise XOR (exclusive OR); then the image edges can be obtained. Therefore, it is worth researching the dynamical behaviors of rule 24 .

In this paper, complex dynamical behaviors of rule 24 are studied in detail. We obtain two conditions according to Bernoulli $\sigma_{\tau}$-shift evolution for attractors under rule 24 . The corresponding strongly connected graphs of transition matrices of determinative block systems are given. In terms of strongly connected subgraphs, we can predict the elements of attractor $\Lambda^{24}$. Finally, we have proven that rule 24 is chaotic in the sense of Li-York and Devaney on sets of $\Lambda^{24}$, which implies that the number of period orbits of rule 24 is infinite.

The rest of the paper is organized as follows. In Section 2, the intent of the paper and notations of symbolic dynamics are introduced. In Section 3, the dynamical behaviors of rule 24 are studied. In Section 4, we prove that four rules of global equivalence $\varepsilon_{5}^{2}$ are topologically conjugate. In Section 5 , characteristic function is used to describe the fact that all points fall into Bernoulli-shift map after two iterations under rule 24 and Lameray diagram is used to show clearly the iterative process of an attractor. Section 6 presents some conclusions.

\section{Preliminaries}

In 1980s, Wolfram proposed CA as models for physical systems which exhibit complex or even chaotic behaviors based on empirical observations, and he divided the 256 elementary cellular automata (ECA) rules informally into four classes using dynamical concepts like periodicity, stability, and chaos [21-23]. However, some authors [24-28] found that some rules of Bernoulli $\sigma_{\tau}$-shift rules are chaotic in the sense of both Li-York and Devaney, where these rules were said to be simple as periodic by Wolfram. Rule 24 is belonging to Bernoulli $\sigma_{\tau}$-shift rules. Therefore, we need to research rule 24 and to find its some new dynamical properties.
In this paper, we will use some notations about CA as follows.

Chua et al. [29] mentioned that each rule has three globally equivalent local rules determined by three corresponding global transformations, namely, left-right transformation $T^{\dagger}$, global complementation $\bar{T}$, and left-right complementation $T^{*}$. Each equivalence class is identified by $\varepsilon_{m}^{\kappa}$, where $\kappa$ is complexity index and $m$ is index of $\kappa$ th class. In [30], the authors presented that 112 rules of 256 local rules were Bernoulli $\sigma_{\tau}$-shift rules. Each of the 112 Bernoulli $\sigma_{\tau}$-shift rules has an ID code $B_{N}[\alpha, \beta, \tau]$, where $\alpha$ denotes the number of attractors of rule $N, \beta$ denotes the slope of the Bernoulli $\sigma_{\tau}$-shift map, and $\tau$ denotes the relevant forward time- $\tau$. Hence, the space-time evolution of any one of the 112 rules on their attractors can be uniquely predicted by two parameters: $\beta= \pm 2^{\sigma}$ and $\tau$. For example, the attractors of rule 14 are $(\beta=-1 / 2, \sigma=-1, \tau=1)$ and $(\beta=2, \sigma=1, \tau=1)$.

Some notations about symbolic dynamics can be referred to in [24-27]

It follows from [31] that the Boolean function of rule 24 is

$$
\left[f_{24}(x)\right]_{i}=\bar{x}_{i-1} \cdot x_{i} \cdot x_{i+1} \oplus x_{i-1} \cdot \bar{x}_{i} \cdot \bar{x}_{i+1},
$$

for all $x \in S^{Z}, i \in Z$, where “.” “-," and “ $\oplus$ ” stand for "AND," "NOT," and "XOR" logical operation, respectively. Sometimes, "." is omitted for simplicity. The truth table of Boolean function of rule 24 is shown in Table 1.

The subset, denoted by $\Lambda^{24}$, is derived from the parameter of rule 24 : $\beta=1 / 2, \sigma=-1, \tau=2$; that is,

$$
\Lambda^{24}=\left\{x \in S^{Z} \mid\left[f_{24}(x)\right]_{i}=x_{i-1}, \forall i \in Z\right\} .
$$

\section{Dynamical Behaviors of $f_{24}$}

In the section, we investigate the complexity and chaotic dynamics of $f_{24}$. In order to give our results, some definitions need be introduced.

Definition 1 (see [32]). A square $\{0,1\}$ matrix $A$ is irreducible, if for every pair of indices $i$ and $j$ there is an $n$ such that $A_{i j}^{n}>$ 0 .

Definition 2 (see [32]). A square $\{0,1\}$ matrix $A$ is aperiodic, if there exists $N$, such that $A_{i j}^{n}>0, n>N$, for all $i, j$.

Definition 3 (see [32]). Suppose that $g: X \rightarrow Y$ is a continuous mapping, where $X$ is a compact topological space. $g$ is said to be topologically mixing if, for any two open sets, $U, V \subset X, \exists N>0$, such that $g^{n}(U) \cap V \neq \emptyset$, for all $n \geq N$.

Definition 4 (see [26]). Let $(X, f)$ and $(Y, g)$ be compact spaces; we say $f$ and $g$ are topologically conjugate if there is a homeomorphism $h: X \rightarrow Y$, such that $h \circ f=g \circ h$.

We give conditions according to Bernoulli $\sigma_{\tau}$-shift evolution for rule 24 as follows.

Theorem 5. For rule 24, there exists a subset $\Lambda^{24} \subset S^{Z}$ which satisfies $\left.f_{24}\right|_{\Lambda^{24}}=\left.\varsigma\right|_{\Lambda^{24}}$ if and only if, for all $x=$ 
$\left(\ldots, x_{-1}, x_{0}, x_{1}, \ldots\right) \in \Lambda^{24}, x_{i-1}, x_{i}$, and $x_{i+1}$ have the following relations.

(i) If $x_{i}=0$, then $x_{i-1}=0, x_{i+1}=0$; $x_{i-1}=0, x_{i+1}=1$, $x_{i+2}=1 ; x_{i-2}=0, x_{i-1}=1, x_{i+1}=0$.

(ii) If $x_{i}=1$, then $x_{i-2}=0, x_{i-1}=0, x_{i+1}=0, x_{i+2}=0$.

Proof. Necessity: suppose that there exists a subset $\Lambda^{24} \in S^{Z}$ such that $\left.f_{24}\right|_{\Lambda^{24}}=\left.\varsigma\right|_{\Lambda^{24}}$. Then for all $x=\left(\ldots, x_{-1}, x_{0}\right.$, $\left.x_{1}, \ldots\right) \in \Lambda^{24}$, we have $\left[f_{24}(x)\right]_{i}=x_{i-1}$, for all $x \in Z$.

(i) If $x_{i}=0$, then $\bar{x}_{i-1} x_{i} x_{i+1} \oplus x_{i-1} \bar{x}_{i} \bar{x}_{i+1}=0 \oplus x_{i-1} \bar{x}_{i+1}$; according to Table 1, we get $x_{i-1}=0, x_{i+1}=0 ; x_{i-1}=$ $0, x_{i+1}=1, x_{i+2}=1 ; x_{i-2}=0, x_{i-1}=1, x_{i+1}=0$.

(ii) If $x_{i}=1$, then $\bar{x}_{i-1} x_{i} x_{i+1} \oplus x_{i-1} \bar{x}_{i} \bar{x}_{i+1}=\bar{x}_{i-1} x_{i+1} \oplus 0$; according to Table 1 , we get $x_{i-2}=0, x_{i-1}=0, x_{i+1}=$ $0, x_{i+2}=0$.

Sufficiency: suppose that there exists a subset $\Lambda^{24} \subset S^{Z}$ and, for all $x \in \Lambda^{24}$, the relations between $x_{i-1}, x_{i}$ and $x_{i+1}$ satisfy the conditions (i) and (ii) in Theorem 5 , for all $i \in Z$.

(i) If $x_{i}=0$, we have $\left[f_{24}(x)\right]_{i}=\bar{x}_{i-1} x_{i} x_{i+1} \oplus x_{i-1} \bar{x}_{i} \bar{x}_{i+1}=$ $0 \oplus x_{i-1} \bar{x}_{i+1}$.

Therefore,

$$
\left[f_{24}(x)\right]_{i}=\left\{\begin{array}{lll}
0, & x_{i-1}=0, & x_{i+1}=0 \\
0, & x_{i-1}=0, & x_{i+1}=1, \\
1, & x_{i-1}=1, & x_{i+1}=0 .
\end{array}\right.
$$

(ii) If $x_{i}=1$, we have

$$
\begin{aligned}
{\left[f_{24}(x)\right]_{i} } & =\bar{x}_{i-1} x_{i} x_{i+1} \oplus x_{i-1} \bar{x}_{i} \bar{x}_{i+1}=\bar{x}_{i-1} x_{i+1} \oplus 0 \\
& =0, x_{i-1}=0, x_{i+1}=0 .
\end{aligned}
$$

Hence, $\left[f_{24}(x)\right]_{i}=x_{i-1}$.

Remark 6. From the definition of subsystem, we know that $\left(\Lambda^{24}, f_{24}\right)$ are subsystems of $\left(S^{Z}, f_{24}\right)$.

The dynamical behaviors of $f_{24}(x)$ on the set $\Lambda^{24}$ are shown as follows.

Let $P=\left\{r_{0}, r_{1}, r_{2}, r_{3}\right\}$ be a new state set, where $r_{0}=(000)$, $r_{1}=(001), r_{2}=(010), r_{3}=(100)$, and $\varpi^{24}=\left\{\left(r r^{\prime}\right) \mid r=\right.$ $\left(b_{0} b_{1} b_{2}\right), r^{\prime}=\left(b_{0}^{\prime} b_{1}^{\prime} b_{2}^{\prime}\right) \in P, \forall 1 \leq j \leq 2$ such that $\left.b_{j}=b_{j-1}^{\prime}\right\}$. Furthermore, subshift $\Lambda_{\omega^{24}}$ of $\varsigma$ is defined as $\Lambda_{\omega^{24}}=\{r=$ $\left.\left(\ldots, r_{-1}, r_{0}, r_{1}, \ldots\right) \in P^{Z} \mid r_{i} \in P, r_{i} r_{i+1} \in \omega^{24}, \forall i \in Z\right\}$. The transition matrix $B^{24}$ of the $\left.\varsigma\right|_{\Lambda_{Q^{24}}}$ is

$$
B^{24}=\left[\begin{array}{llll}
1 & 1 & 0 & 0 \\
0 & 0 & 1 & 0 \\
0 & 0 & 0 & 1 \\
1 & 1 & 0 & 0
\end{array}\right] .
$$

Obviously, $B^{24}$ is a square $\{0,1\}$ matrix. A square $\{0,1\}$ matrix corresponds to a directed graph. The vertices of the graph are the indices for the rows and columns of $B^{24}$. There

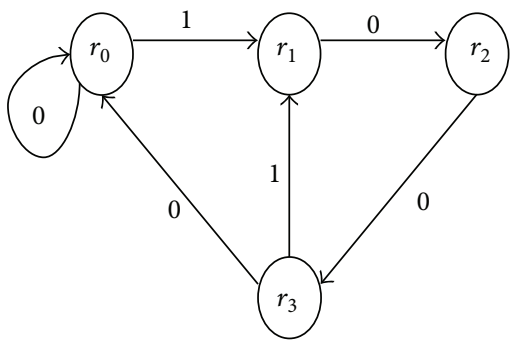

FIGURE 1: The corresponding graph $G^{24}$ of the matrix $B^{24}$.

is an edge from vertex $i$ to vertex $j$ if $B_{i j}^{24}=1$. A square $\{0,1\}$ matrix is irreducible if and only if the corresponding graph is strongly connected. If $\Lambda_{A}$ is a two-order subshift of finite type, then it is topologically mixing if and only if $A$ is irreducible and aperiodic.

We give corresponding graph $G^{24}$ of the matrix $B^{24}$ in Figure 1, where vertices are the elements of set $P$. It is obvious that $G^{24}$ is a strongly connected graph.

Remark 7. By definition, we know that $P$ is the determinative block system of $\Lambda^{24}$ and $\Lambda^{24}=\Lambda_{P}^{24}$ is a subshift of finite type.

Remark 8. Carefully observing Figure 1 , we find that there are several strongly connected subgraphs: $r_{0} \rightarrow r_{0}, r_{1} \rightarrow r_{2} \rightarrow$ $r_{3} \rightarrow r_{1}, r_{0} \rightarrow r_{1} \rightarrow r_{2} \rightarrow r_{3} \rightarrow r_{0}$. The elements of $\Lambda_{P}^{24}$ will be composed by all vertices of subgraphs, respectively. For example, $x \in \Lambda_{P}^{24}$ and $x_{1}$ is composed of vertices of subgraph $r_{1} \rightarrow r_{2} \rightarrow r_{3} \rightarrow r_{1}$; then we have $r_{0} \nless x_{1}$ and vertices of subgraph $r_{1} \rightarrow r_{2} \rightarrow r_{3}$ will appear in $x_{1}$, if $\left|x_{1}\right|=3 k, k=1,2 \ldots$

Remark 9. Let $e_{1}=$ (011), $e_{2}=$ (101), $e_{3}=$ (110), and $e_{4}=(111)$. In terms of Table 1 , we find that, after one iteration, the possible sequences are generated by $e_{1}, e_{2}, e_{3}$, and $e_{4}$ as follows:

$$
\begin{aligned}
& e_{1} \rightarrow 010, e_{2} \rightarrow 000 \text { or } 001, e_{3} \rightarrow 000,001,100 \text { or } \\
& 101, e_{4} \rightarrow 000 \text { or } 100 .
\end{aligned}
$$

We find that, though $e_{1}, e_{2}, e_{3}, e_{4} \nless \Lambda_{P}^{24}$, after one or two iterations, they belong to $\Lambda_{P}^{24}$. Therefore, we guess that $\Lambda_{P}^{24}$ is the global attractor of $f_{24}$.

Theorem 10. $\Lambda_{P}^{24}$ is global attractor of $f_{24}$.

Proof. To prove that $\Lambda_{P}^{24}$ is the global attractor of $f_{24}$, we consider two situations.

(i) $x \in \Lambda_{P}^{24}$. Since $\Lambda_{P}^{24}$ is invariant under $f_{24}$, we have $f_{24}(x) \in \Lambda_{P}^{24}$.

(ii) $x \in S^{Z}-\Lambda_{P}^{24}$. Suppose that there exist $y \in S^{Z}-\Lambda_{P}^{24}$ such that $f_{24}(x)=y$. Then, we have $(011) \prec y,(101) \prec y$, $(110) \prec y$, or $(111) \prec y$ by Theorem 5 . Without loss of generality, we consider several situations as follows. 
(a) Let $y_{[-1,1]}=(011)$. Since $y_{i}=\left[f_{24}(x)\right]_{i}$, for all $i \in Z$, we especially have

$$
\begin{gathered}
{\left[f_{24}(x)\right]_{-1}=\bar{x}_{-2} \cdot x_{-1} \cdot x_{0} \oplus x_{-2} \cdot \bar{x}_{-1} \cdot \bar{x}_{0}=0,} \\
{\left[f_{24}(x)\right]_{0}=\bar{x}_{-1} \cdot x_{0} \cdot x_{1} \oplus x_{-1} \cdot \bar{x}_{0} \cdot \bar{x}_{1}=1,} \\
{\left[f_{24}(x)\right]_{1}=\bar{x}_{0} \cdot x_{1} \cdot x_{2} \oplus x_{0} \cdot \bar{x}_{1} \cdot \bar{x}_{2}=1 .}
\end{gathered}
$$

Therefore, it follows from Table 1 that $x_{[-1,1]} \in\{(011),(100)\}$ by (7), which implies that $\bar{x}_{0} \cdot x_{1} \cdot x_{2} \oplus x_{0} \cdot \bar{x}_{1} \cdot \bar{x}_{2}=0$. It is in contradiction to $(8)$. Hence, $y_{[-i, i]}=(\ldots, 0,1,1, \ldots)$ is no ancestor.

(b) Let $y_{[-1,1]}=(101)$. Since $y_{i}=\left[f_{24}(x)\right]_{i}$, for all $i \in Z$, we especially have

$$
\begin{gathered}
{\left[f_{24}(x)\right]_{-1}=\bar{x}_{-2} \cdot x_{-1} \cdot x_{0} \oplus x_{-2} \cdot \bar{x}_{-1} \cdot \bar{x}_{0}=1,} \\
{\left[f_{24}(x)\right]_{0}=\bar{x}_{-1} \cdot x_{0} \cdot x_{1} \oplus x_{-1} \cdot \bar{x}_{0} \cdot \bar{x}_{1}=0,} \\
{\left[f_{24}(x)\right]_{1}=\bar{x}_{0} \cdot x_{1} \cdot x_{2} \oplus x_{0} \cdot \bar{x}_{1} \cdot \bar{x}_{2}=1 .}
\end{gathered}
$$

Therefore, it follows from Table 1 that $x_{[-2,0]} \in$ $\{(011),(100)\}$ by (9). Furthermore, if $x_{[-2,0]}=(011)$, we know that $y_{[-i, i]}=(\ldots, 0,1,1, \ldots)$ is no ancestor. If $x_{[-2,0]}=(100)$, then only (011) satisfies (11). However, we know that $y_{[-i, i]}=(\ldots, 0,1,1, \ldots)$ is no ancestor. The situations of (110) and (111) are similar to those of (011) or (101). In conclusion, if $x \in S^{Z}-\Lambda_{P}^{24}, x$ will be no ancestor or its ancestor will be no ancestor. Therefore, $\Lambda_{P}^{24}$ is global attractor of $f_{24}$.

Remark 11. From the above conclusion, we find that all binary sequences will be bound to fall into $\Lambda_{P}^{24}$ after two iterations under $f_{24}$.

Based on the above definitions and analysis, we have the following results.

Theorem 12. (a) $\varsigma: \Lambda_{P}^{24} \rightarrow \Lambda_{P}^{24}$ and $\varsigma: \Lambda_{\omega^{24}} \rightarrow \Lambda_{\omega^{24}}$ are topologically conjugate.

(b) $\varsigma: \Lambda_{P}^{24} \rightarrow \Lambda_{P}^{24}$ is topologically mixing.

(c) $f_{24}: \Lambda_{P}^{24} \rightarrow \Lambda_{P}^{24}$ is topologically mixing.

(d) The topological entropy ent $\left(\left.f_{24}\right|_{\Lambda_{P}^{24}}\right)=\operatorname{ent}\left(\left.\varsigma\right|_{\Lambda_{P}^{24}}\right)=$ 0.3823 .

Proof. (a) We find out a homeomorphism $h$ from $\Lambda_{\lambda_{1}^{24}}$ to $\Lambda_{\omega_{1}^{24}}$.

Define

$$
\begin{aligned}
h: \Lambda_{P}^{24} & \longrightarrow \Lambda_{\omega^{24}}, \\
x=\left(\ldots, x_{-1}, x_{0}^{*}, x_{1}, \ldots\right) & \longmapsto\left(\ldots, r_{-1}, r_{0}^{*}, r_{1}, \ldots\right),
\end{aligned}
$$

where $r_{i}=\left(x_{i} x_{i+1} x_{i+2}\right)$, for all $i \in Z$. In fact, by the definition of $\Lambda_{\omega^{24}}$, we have $h(x) \in \Lambda_{\omega^{24}}$, for all $x \in \Lambda_{P}^{24}$; thus $h\left(\Lambda_{P}^{24}\right) \subseteq$ $h\left(\Lambda_{\omega^{24}}\right)$. Then, it is easy to check that $h$ is homeomorphism and $\left.\left.h \circ \varsigma\right|_{\Lambda_{P}^{24}} \rightarrow \varsigma\right|_{\Lambda_{\omega^{24}}} \circ h$. Therefore, $\left.\varsigma\right|_{\Lambda_{P}^{24}}$ and $\left.\varsigma\right|_{\Lambda_{\omega^{24}}}$ are topologically conjugate. (b) Since $\left.\varsigma\right|_{\Lambda_{P}^{24}}$ and $\left.\varsigma\right|_{\Lambda_{\Phi^{24}}}$ are topologically conjugate, we only need to check that the transition matrix $B^{24}$ of $\left.\varsigma\right|_{\Lambda_{\omega^{24}}}$ is irreducible and aperiodic. Actually, $\left(B^{24}\right)^{n}>0$, for all $n \geq 5$. By Definitions 1 and 2 , we know that $B^{24}$ is irreducible and aperiodic. So, in terms of $[32,33], \varsigma: \Lambda_{P}^{24} \rightarrow \Lambda_{P}^{24}$ is topologically mixing.

(c) Since $f_{24}(x)$ and $\varsigma(x)$ are equal in the set $\Lambda_{P}^{24}$ and $\left.\varsigma\right|_{\Lambda_{P}^{24}}$ is topologically mixing, $\left.f_{24}\right|_{\Lambda_{P}^{24}}$ is topologically mixing.

(d) The topological entropy $\varsigma$ on $\Lambda_{P}^{24}$ equals $\log \rho\left(B^{24}\right)$, where $\rho\left(B^{24}\right)$ is the spectral radius of the transition matrix $B^{24}$ of the subshift $\Lambda_{P}^{24}$. So, $\operatorname{ent}\left(\left.\varsigma\right|_{\Lambda_{P}^{24}}\right)=\log \rho\left(B^{24}\right)=$ 0.3823 . Because two topological conjugate systems have the same topological entropy, the topological entropy of $\left.f_{24}\right|_{\Lambda_{P}^{24}}$ is equal to that of $\left.\varsigma\right|_{\Lambda_{P}^{24}}$; namely, $\operatorname{ent}\left(\left.f_{24}\right|_{\Lambda_{P}^{24}}\right)=\operatorname{ent}\left(\left.\varsigma\right|_{\Lambda_{P}^{24}}\right)=$ 0.3823 .

Theorem 13. $f_{24}$ is chaotic in the sense of both Li-Yorke and Devaney on $\Lambda_{P}^{24}$.

Proof. It follows from [33] that the positive topological entropy implies chaos in the sense of Li-Yorke and topologically mixing implies chaos in the sense of Li-Yorke and Devaney, since rule $N=24$ possesses very rich and complicated dynamical properties on $\Lambda_{P}^{24}$.

\section{The Relationship between Four Rules of Global Equivalence Class $\varepsilon_{5}^{2}$}

In this section, we will discuss the relationship between four rules of global equivalence class $\varepsilon_{5}^{2}$. In [31], rules $24,66,231$, and 189 are partitioned into global equivalence class $\varepsilon_{5}^{2}$. Next, we prove that they are topologically conjugate to each other.

Theorem 14. (i) $f_{24}: S^{Z} \rightarrow S^{Z}$ and $f_{66}: S^{Z} \rightarrow S^{Z}$ are topologically conjugate.

(ii) $f_{24}: S^{Z} \rightarrow S^{Z}$ and $f_{231}: S^{Z} \rightarrow S^{Z}$ are topologically conjugate.

(iii) $f_{24}: S^{Z} \rightarrow S^{Z}$ and $f_{189}: S^{Z} \rightarrow S^{Z}$ are topologically conjugate.

Proof. By [31], we have

$$
\begin{aligned}
& f_{24}=\bar{x}_{i-1} x_{i} x_{i+1} \oplus x_{i-1} \bar{x}_{i} \bar{x}_{i+1}, \\
& f_{66}=x_{i-1} x_{i} \bar{x}_{i+1} \oplus \bar{x}_{i-1} \bar{x}_{i} x_{i+1}, \\
& f_{231}=\overline{x_{i-1} \bar{x}_{i} \bar{x}_{i+1} \oplus \bar{x}_{i-1} x_{i} x_{i+1}}, \\
& f_{189}=\overline{\bar{x}_{i-1} \bar{x}_{i} x_{i+1} \oplus x_{i-1} x_{i} \bar{x}_{i+1}} .
\end{aligned}
$$

Then we have

$$
\begin{aligned}
& T^{\dagger} \circ f_{24}\left(x_{i}\right)=\bar{x}_{-i+1} x_{-i} x_{-i-1} \oplus x_{-i+1} \bar{x}_{-i} \bar{x}_{-i-1}, \\
& f_{66} \circ T^{\dagger}\left(x_{i}\right)=x_{-i-1} x_{-i} \bar{x}_{-i+1} \oplus \bar{x}_{-i-1} \bar{x}_{-i} x_{-i+1},
\end{aligned}
$$




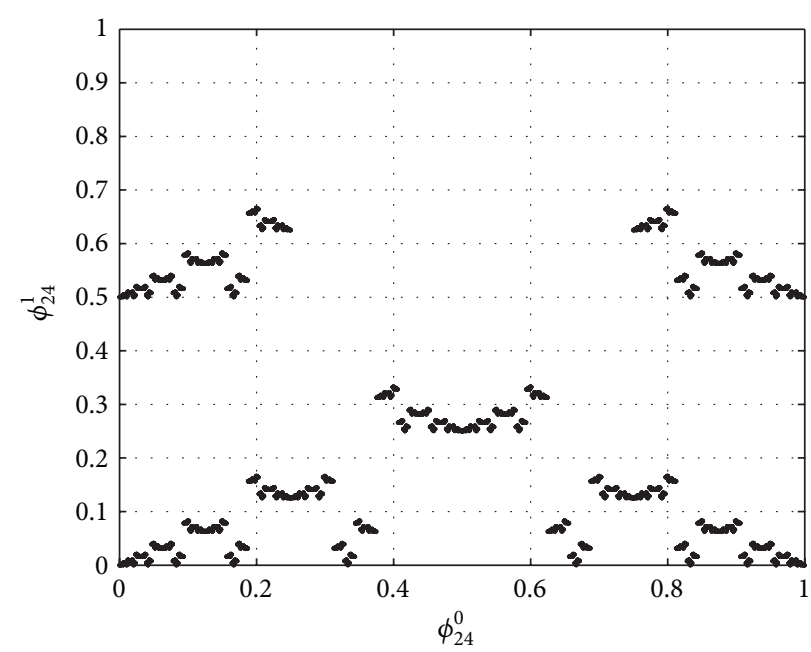

(a)

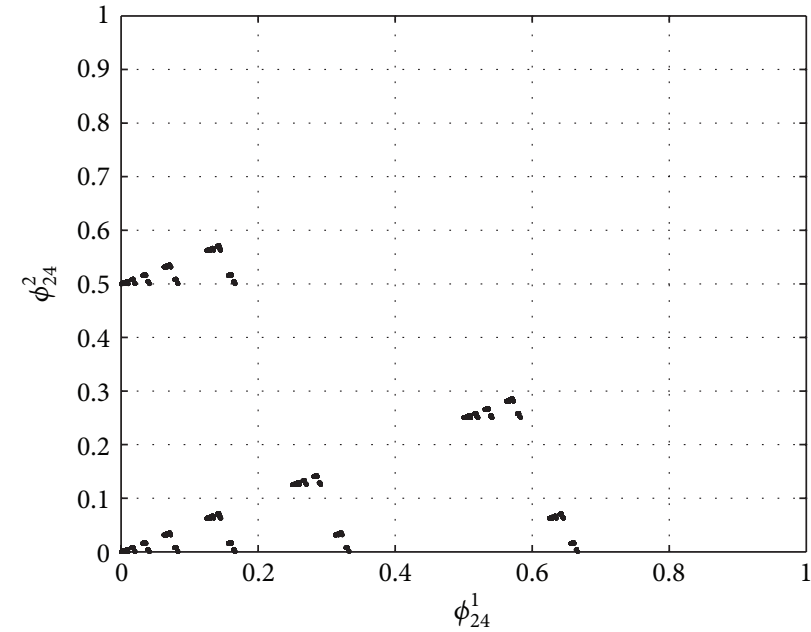

(b)

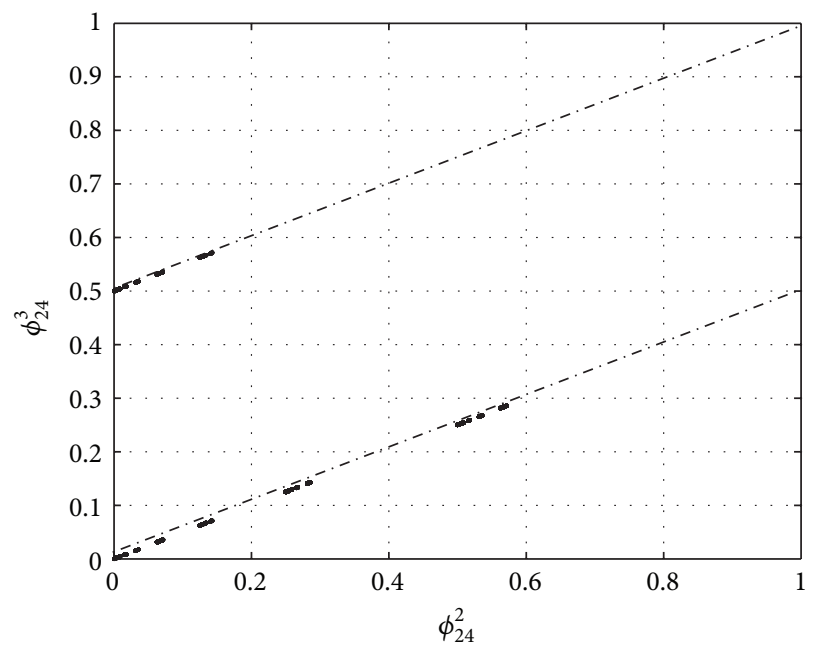

(c)

FIGURE 2: Let $I=14$. (a), (b), and (c) describe the process, where all points fall into Bernoulli-shift map after two iterations under rule 24.

$$
\begin{aligned}
& \bar{T} \circ f_{24}\left(x_{i}\right)=\overline{\bar{x}_{i-1} x_{i} x_{i+1} \oplus x_{i-1} \bar{x}_{i} \bar{x}_{i+1}}, \\
& f_{231} \circ \bar{T}\left(x_{i}\right)=\overline{\bar{x}_{i-1} x_{i} x_{i+1} \oplus x_{i-1} \bar{x}_{i} \bar{x}_{i+1}} \text {, } \\
& \bar{T} \circ T^{\dagger} \circ f_{24}\left(x_{i}\right)=\overline{\bar{x}}_{-i+1} x_{-i} x_{-i-1} \oplus x_{-i+1} \bar{x}_{-i} \bar{x}_{-i-1} \text {, } \\
& f_{189} \circ \bar{T} \circ T^{\dagger}\left(x_{i}\right)=\overline{x_{-i-1} x_{-i} \bar{x}_{-i+1} \oplus \bar{x}_{-i-1} \bar{x}_{-i} x_{-i+1}} \text {. }
\end{aligned}
$$

Therefore, we have $T^{\dagger} \circ f_{24}\left(x_{i}\right)=f_{66} \circ T^{\dagger}\left(x_{i}\right), \bar{T} \circ f_{24}\left(x_{i}\right)=$ $f_{231} \circ T^{\dagger}\left(x_{i}\right), \bar{T} \circ T^{\dagger} \circ f_{24}\left(x_{i}\right)=f_{189} \circ \bar{T} \circ T^{\dagger}\left(x_{i}\right)$. So, $f_{24}, f_{66}$, $f_{231}$, and $f_{189}$ are topologically conjugate to each other.

Remark 15. If there are two systems topologically conjugate, these two systems have the same dynamical properties. Rules $f_{24}, f_{66}, f_{231}$, and $f_{189}$ are topologically conjugate, respectively. Therefore, if we know that one of four rules is chaotic in the sense of both Li-Yorke and Devaney in its attractors, we can deem that the other four rules are chaotic in the sense of both Li-Yorke and Devaney in their attractors, respectively.

\section{Using Diagrams to Explain Attractors of Four Rules}

First, we give a definition on global characteristic function [30]. Given any local rule $N$ and binary configuration, $x=$ $\left[x_{0} x_{1} \cdots x_{I-1} x_{I}\right]$ for a ECA, where $x_{i} \in\{0,1\}$. Then we can uniquely associate the Boolean string $x$ with the binary expansion of a real number $0 . x_{0} x_{1} \cdots x_{I-1} x_{I}$ on the unit interval $[0,1]$ :

$$
x=\left[x_{0} x_{1} \cdots x_{I-1} x_{I}\right] \longmapsto \phi \triangleq 0 . x_{0} x_{1} \cdots x_{I-1} x_{I},
$$

where $\phi=\sum_{i=0}^{I} 2^{-(i+1)} x_{i}$ is the decimal form of Boolean string $x=\left[x_{0} x_{1} \cdots x_{I-1} x_{I}\right]$. The ECA' characteristic function $\chi_{N}$ of rule $N$ is defined as

$$
\chi_{N}: Q[0,1] \longrightarrow Q[0,1], \quad \text { that is, } \phi_{n}^{1}=\chi_{N}^{1}\left(\phi_{n-1}\right),
$$

where $Q$ denotes rational numbers. 


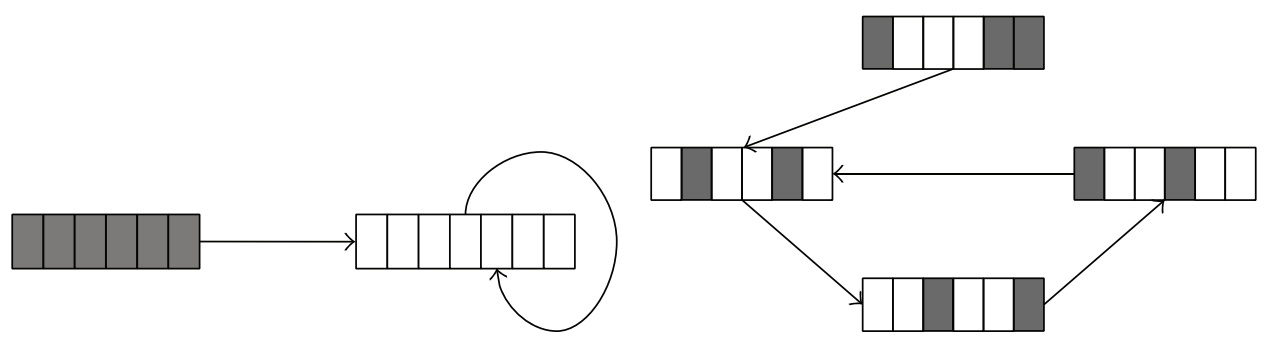

(a)

(b)

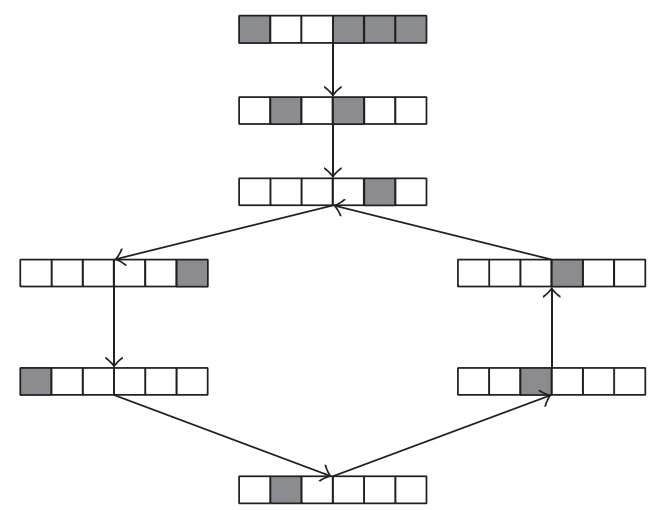

(c)

FIgURE 3: Several attractors of rule 24, where $I=5$, and the white lattice stands for 0 and the black for 1.

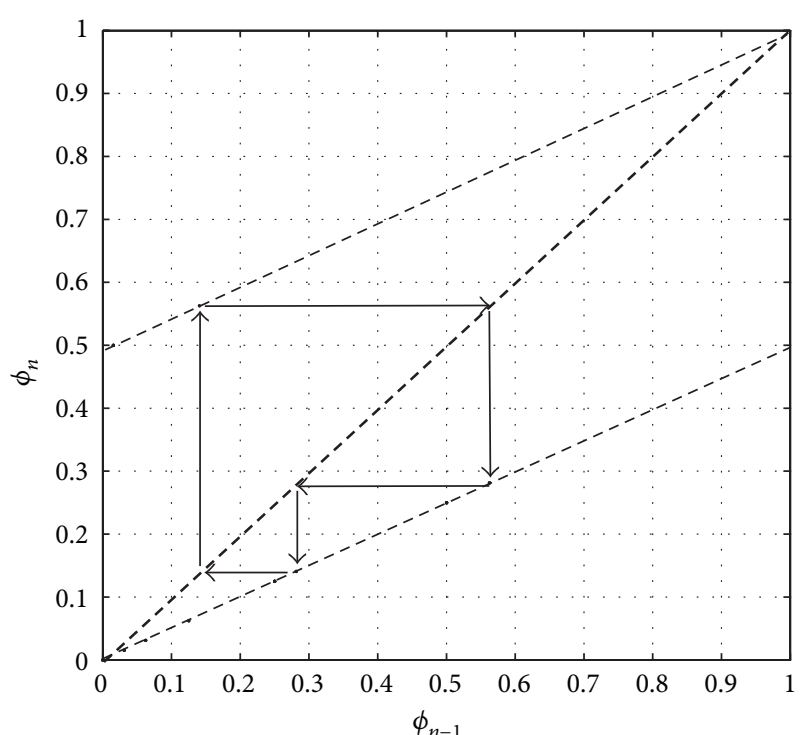

FIGURE 4: The evolution of characteristic function of the period-3 attractor, where the values of characteristic function of the attractor are $0.2813,0.1406$, and 0.5625 , respectively.

We choose $I=14$. Figure 2 shows characteristic functions of rule 24. Figures 2(a), 2(b), and 2(c) describe the fact that all points fall into Bernoulli-shift map after two iterations under rule 24, which shows that $\Lambda_{P}^{24}$ is global attractor of $f_{24}$. The phenomenon also shows that the forecast in Remark 11 is correct.
If we choose different values of $I$ for rule 24 , we can get different initial binary configurations for the evolution of rule 24. The different initial binary configurations may lead to different attractor periods. If the value of $I$ is fixed, we may find the periods of attractors different. Given $I=5$, we find three attractors of rule 24 shown in Figures 3(a), 3(b), and 3 (c). Periods of these attractors are 1, 3, and 6, respectively.

Remark 16. The sequence $x=\left[x_{0} x_{1} \cdots x_{I-1} x_{I}\right]$ consists of arbitrary alternations of 0 and 1 , and there are $2^{(I+1)}$ different possibilities of choice for $x$.

Next, we use Lameray diagram [34] to present the evolution process of attractor. In terms of the attractor of Figure 3(b), we get that the values of characteristic function of the attractor are $0.2813,0.1406$, and 0.5625 , respectively. Figure 4 shows the iterative process of the attractor. Then, we can associate the period-3 attractor of rule 24 as a period-3 point of a continuous map $f:[0,1] \rightarrow[0,1]$ which we know to be chaotic because "period-3 implies chaos" [35].

\section{Conclusion}

In this work, we characterize the global attractor of rule 24 . We derive the conditions according to Bernoulli $\sigma_{\tau}$-shift evolution for attractor of rule 24 . Then, in terms of the transition matrix of determinative block system of subsystem of rule 24, we obtain the value of topological entropy of subsystem. By corresponding strongly connected graph of transition matrix of determinative block system of subsystem $\Lambda^{24}$, we guess that $\Lambda_{P}^{24}$ is the global attractor of $f_{24}$. Furthermore, we prove 
that $\Lambda_{P}^{24}$ is the global attractor of $f_{24}$. We find that rule 24 is topologically mixing on $\Lambda_{P}^{24}$. Then, we prove that four rules of global equivalence $\varepsilon_{5}^{2}$ of ECA are topologically conjugate. So, these four rules are chaotic in the sense of both Li-Yorke and Devaney on their attractors, respectively. We use diagrams to explain the attractor of rule 24 , where characteristic function and Lameray diagram are used to describe the fact that all points fall into Bernoulli-shift map after two iterations and to show clearly the iterative process of an attractor, respectively.

\section{Conflict of Interests}

The authors declare that there is no conflict of interests regarding the publication of this paper.

\section{Acknowledgments}

This work was supported in part by the Research Project of Chongqing University of Science and Technology (CK2013B15), Scientific and Technological Research Program of Chongqing Municipal Education Commission (KJ131401, KJ131416), the Natural Science Foundation Project of CQCSTC (cstc2012jjB0095), the National Natural Science Foundation of China (51275547), and Achievement Transfer Program of Institutions of Higher Education in Chongqing (KJ121413).

\section{References}

[1] J. von Neumann, The General and Logical Theory of Automata, Pergamon Press, Lendon, UK, 1951.

[2] H. Beigy and M. R. Meybodi, "Cellular learning automata with multiple learning automata in each cell and its applications," IEEE Transactions on Systems, Man, and Cybernetics, Part B, vol. 40, no. 1, pp. 54-65, 2010.

[3] W. Pries, A. Thanailakis, and H. C. Card, "Group properties of cellular automata and VLSI applications," IEEE Transactions on Computers, vol. C-35, no. 12, pp. 1013-1024, 1986.

[4] Q.-X. Liu, Z. Jin, and M.-X. Liu, "Spatial organization and evolution period of the epidemic model using cellular automata," Physical Review E, vol. 74, no. 3, Article ID 031110, 6 pages, 2006.

[5] G.-Q. Sun, Z. Jin, and L. Li, "Emergent turing pattern in epidemic spreading using cellular automaton," International Journal of Modern Physics B, vol. 25, no. 32, pp. 4605-4613, 2011.

[6] G.-Q. Sun, Z. Jin, L.-P. Song, A. Chakraborty, and B.-L. Li, "Phase transition in spatial epidemics using cellular automata with noise," Ecological Research, vol. 26, no. 2, pp. 333-340, 2011.

[7] S. Nandi, B. K. Kar, and P. Pal Chaudhuri, "Theory and applications of cellular automata in cryptography," IEEE Transactions on Computers, vol. 43, no. 12, pp. 1346-1357, 1994.

[8] A. Abdo, S. Lian, I. Ismail, M. Amin, and H. Diab, "A cryptosystem based on elementary cellular automata," Communications in Nonlinear Science and Numerical Simulation, vol. 18, no. 1, pp. 136-147, 2013.

[9] J.-C. Jeon and K.-Y. Yoo, "Elliptic curve based hardware architecture using cellular automata," Mathematics and Computers in Simulation, vol. 79, no. 4, pp. 1197-1203, 2008.

[10] Z. Eslami, S. H. Razzaghi, and J. Z. Ahmadabadi, "Secret image sharing based on cellular automata and steganography," Pattern Recognition, vol. 43, no. 1, pp. 397-404, 2010.
[11] R.-J. Chen and S.-J. Horng, "Novel SCAN-CA-based image security system using SCAN and 2-D von Neumann cellular automata," Signal Processing: Image Communication, vol. 25, no. 6, pp. 413-426, 2010.

[12] L. Feng, X. Liao, Q. Han, and L. Song, "Modeling and analysis of peer-to-peer botnets," Discrete Dynamics in Nature and Society, vol. 2012, Article ID 865075, 18 pages, 2012.

[13] L. Feng, X. Liao, Q. Han, and H. Li, "Dynamical analysis and control strategies on malware propagation model," Applied Mathematical Modelling, vol. 37, no. 16-17, pp. 8225-8236, 2013.

[14] L.-P. Song, Z. Jin, G.-Q. Sun, J. Zhang, and X. Han, "Influence of removable devices on computer worms: dynamic analysis and control strategies," Computers \& Mathematics with Applications, vol. 61, no. 7, pp. 1823-1829, 2011.

[15] W. M. Van Ballegooijen and M. C. Boerlijst, "Emergent tradeoffs and selection for outbreak frequency in spatial epidemics," Proceedings of the National Academy of Sciences of the United States of America, vol. 101, no. 52, pp. 18246-18250, 2004.

[16] G.-Q. Sun, Q.-X. Liu, Z. Jin, A. Chakraborty, and B.-L. Li, "Influence of infection rate and migration on extinction of disease in spatial epidemics," Journal of Theoretical Biology, vol. 264, no. 1, pp. 95-103, 2010.

[17] Q. Han, C. D. Li, and J. J. Huang, "Anticipating synchronization of chaotic systems with time delay and parameter mismatch," Chaos, vol. 19, Article ID 013104, 10 pages, 2009.

[18] Q. Han, C. Li, and T. Huang, "Anticipating synchronization of a class of chaotic systems," Chaos, vol. 19, no. 2, Article ID 023105, 10 pages, 2009.

[19] F. Bao, "Cryptanalysis of a partially known cellular automata cryptosystem," IEEE Transactions on Computers, vol. 53, no. 11, pp. 1493-1497, 2004.

[20] M. Tomassini and M. Perrenoud, "Cryptography withcellular automata," Applied Soft Computing, vol. 1, no. 2, pp. 151-160, 2001.

[21] S. Wolfram, "Universality and complexity in cellular automata," Physica D, vol. 10, no. 1-2, pp. 1-35, 1984.

[22] S. Wolfram, Theory and Applications of Cellular Automata, vol. 1 of Advanced Series on Complex Systems, World Scientific Publishing, Singapore, 1986.

[23] S. Wolfram, A New Kind of Science, Wolfram Media, Champaign, Ill, USA, 2002.

[24] F.-Y. Chen, W.-F. Jin, G.-R. Chen, F.-F. Chen, and L. Chen, "Chaos of elementary cellular automata rule 42 of Wolfram's class II," Chaos, vol. 19, no. 1, Article ID 013140, 6 pages, 2009.

[25] F.-F. Chen and F.-Y. Chen, "Complex dynamics of cellular automata rule 119," Physica A, vol. 388, no. 6, pp. 984-990, 2009.

[26] L. Chen, F. Y. Chen, F. Chen, and W. Jin, "Complex symbolic dynamics of bernoulli shift cellular automata rule," in Proceedings of the 9th International Conference for Young Computer Scientists (ICYCS '08), pp. 2868-2873, November 2008.

[27] Q. Han, X. Liao, C. Li, and L. Feng, "Complex dynamics behaviors in cellular automata rule 35," Journal of Cellular Automata, vol. 6, no. 6, pp. 487-504, 2011.

[28] Q. Han, X. Liao, and C. Li, "Complex dynamic behaviors in cellular automata rule 14," Discrete Dynamics in Nature and Society, Article ID 258309, 12 pages, 2012.

[29] L. O. Chua, V. I. Sbitnev, and S. Yoon, "A nonlinear dynamics perspective of Wolfram's new kind of science. III. Predicting the unpredictable," International Journal of Bifurcation and Chaos, vol. 14, no. 11, pp. 3689-3820, 2004. 
[30] L. O. Chua, V. I. Sbitnev, and S. Yoon, "A nonlinear dynamics perspective of Wolfram's new kind of science. IV. From Bernoulli shift to $1 / f$ spectrum," International Journal of Bifurcation and Chaos, vol. 15, no. 4, pp. 1045-1183, 2005.

[31] J. Guan, S. Shen, C. Tang, and F. Chen, "Extending Chua's global equivalence theorem on Wolfram's new kind of science," International Journal of Bifurcation and Chaos, vol. 17, no. 12, pp. 4245-4259, 2007.

[32] B. P. Kitchens, Symbolic Dynamics: One-Sided, Two-Sided and Countable State Markov Shifts, Universitext, Springer, Berlin, Germany, 1998.

[33] Z. Zhou, Symbolic Dynamics, Shanghai Scientific and Technological Education Publishing House, Shanghai, China, 1997, (Chinese).

[34] L. P. Shilnikov, A. L. Shilnikov, D. V. Turaev, and L. O. Chua, Methods of Qualitative Theory in Nonlinear Dynamics. Part I, vol. 4 of World Scientific Series on Nonlinear Science. Series A: Monographs and Treatises, World Scientific Publishing, River Edge, NJ, USA, 1998.

[35] T. Y. Li and J. A. Yorke, "Period three implies chaos," The American Mathematical Monthly, vol. 82, no. 10, pp. 985-992, 1975. 


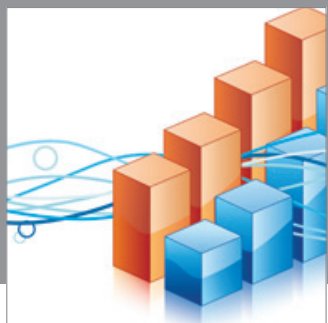

Advances in

Operations Research

mansans

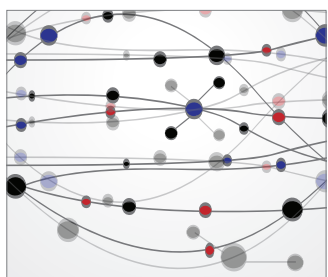

The Scientific World Journal
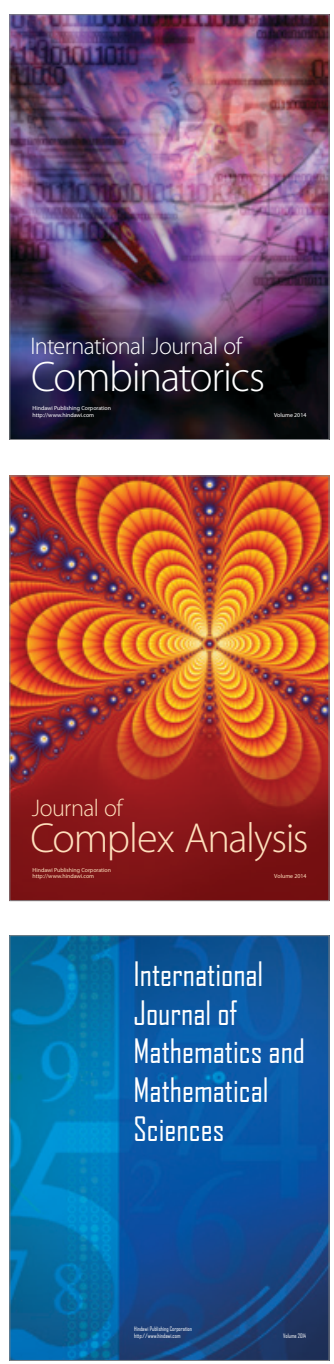
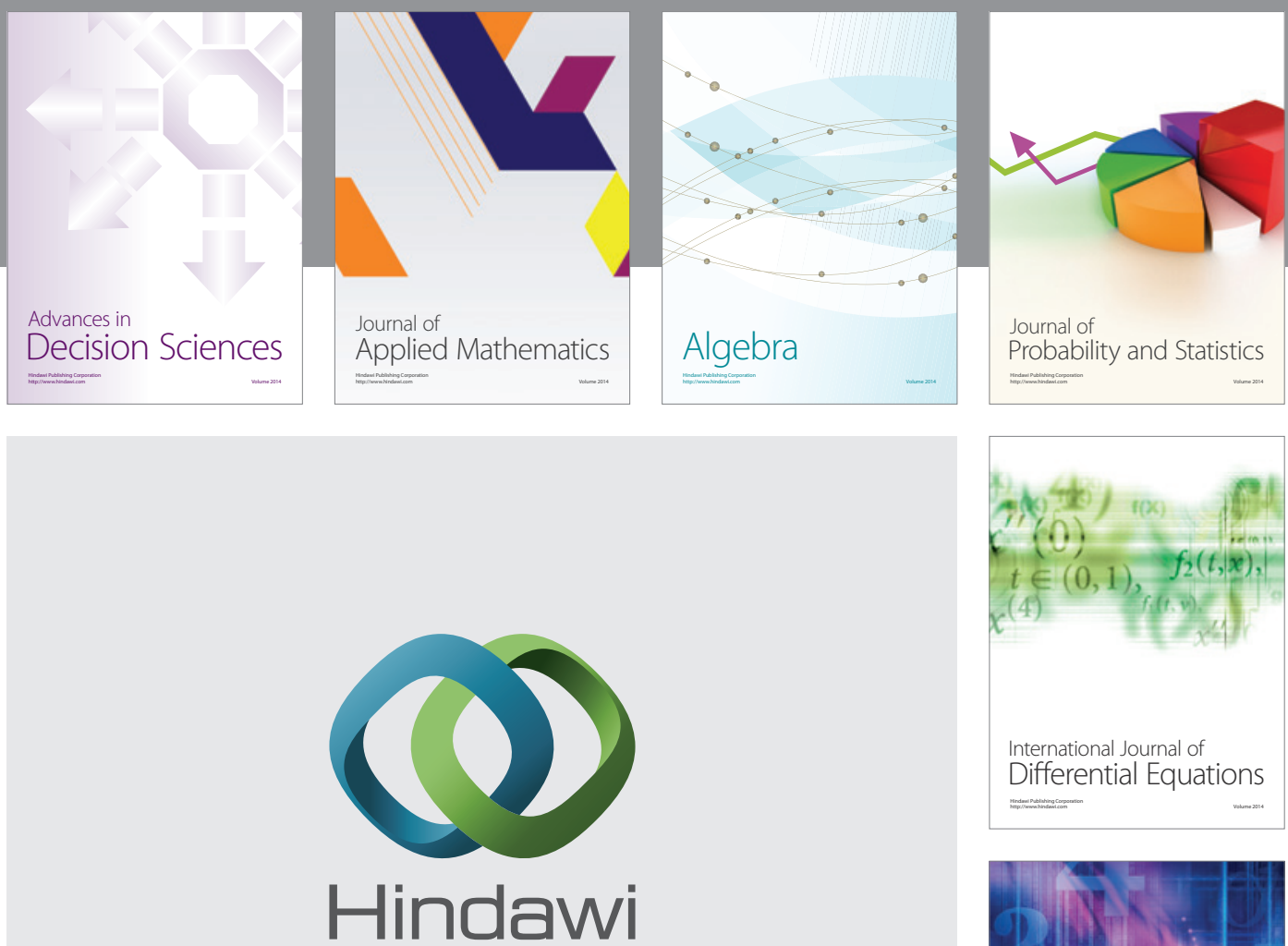

Submit your manuscripts at http://www.hindawi.com
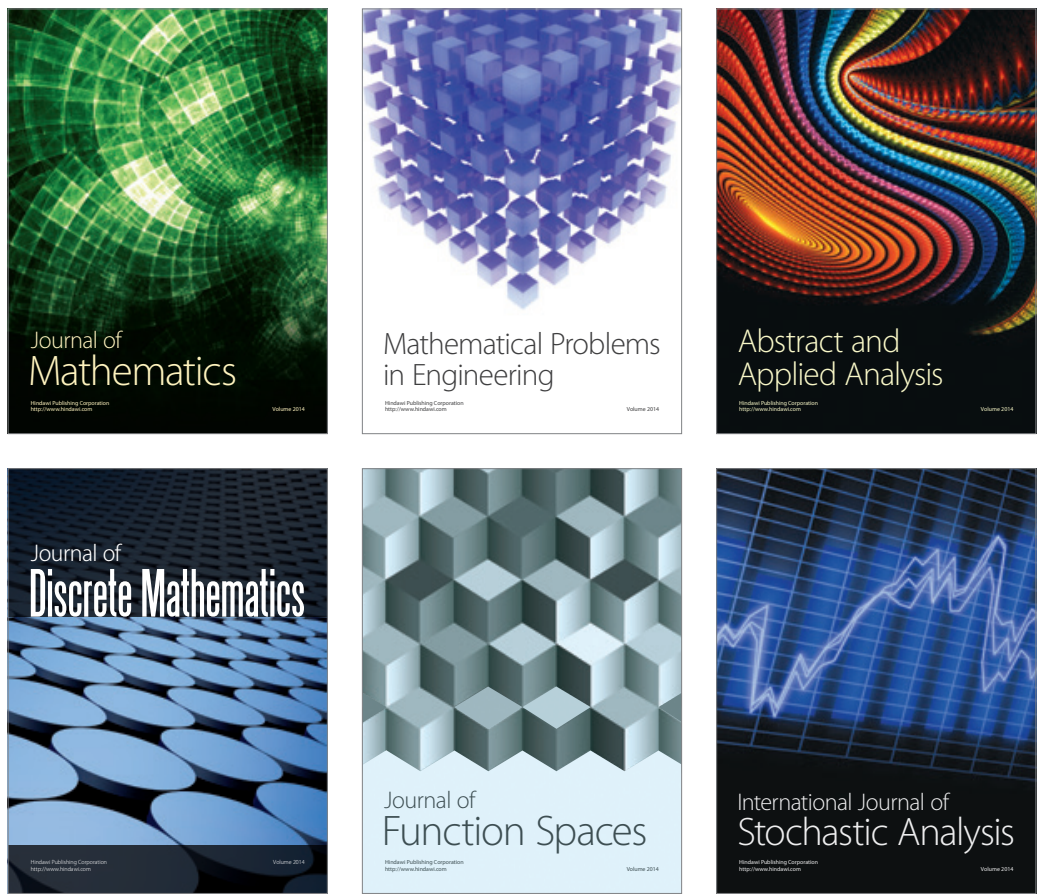

Journal of

Function Spaces

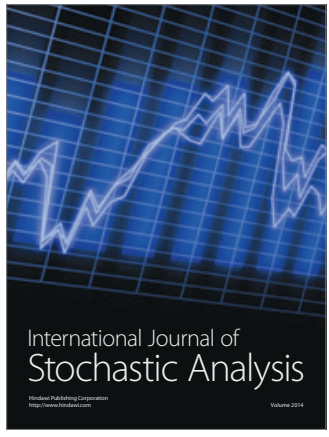

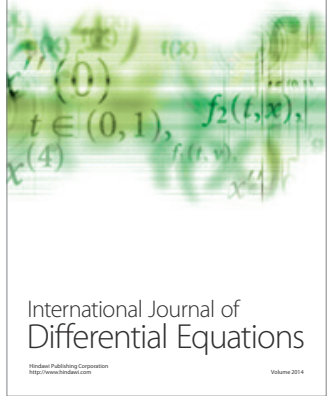
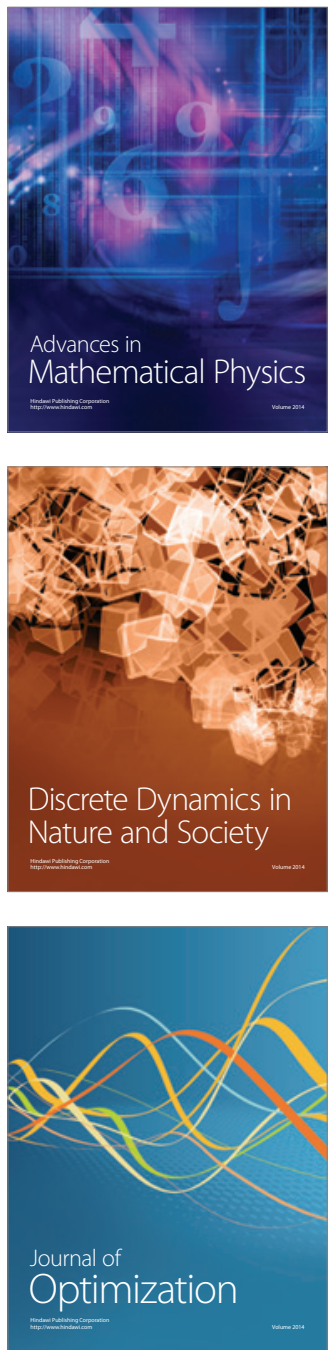